\title{
Exclusive Breastfeeding Practice And Associated Factors Among Employed And Unemployed Mothers In Ethiopia: Comparative Cross Sectional Study Using EDHS 2016
}

Desta Markos Minamo ( $\sim$ destmark11@gmail.com )

Research article

Keywords: Exclusive breastfeeding, maternal employment, EDHS, Ethiopia

Posted Date: October 19th, 2021

DOI: https://doi.org/10.21203/rs.3.rs-989840/v1

License: (a) This work is licensed under a Creative Commons Attribution 4.0 International License.

Read Full License 
EXCLUSIVE BREASTFEEDING PRACTICE AND ASSOCIATED FACTORS AMONG EMPLOYED AND UNEMPLOYED MOTHERS IN ETHIOPIA:

\section{COMPARATIVE CROSS SECTIONAL STUDY USING EDHS 2016}

\section{DESTA MARKOS, ${ }^{1}$ ESHETU YISIKI ${ }^{2}$}

$$
{ }^{1} \text { Lecturer at school of public health, Arba minch university }
$$$$
{ }^{2} \text { Lecturers at department of midwifery, Arba minch university }
$$

\section{${ }^{1}$ Desta Markos: destmark11@gmail.com}

${ }^{2}$ Eshetu Yisihak : eshetuyisihak@gmail.com

*Corresponding author: Desta Markos, school of public health, College of Medicine and Health Sciences, Arba Minch University, Arba Minch, Ethiopia

12 Phone number: +251916481409, gmail, destmark11@gmail.com

Postal code: 21

Introduction: Contrary to the recommendation, children under the age of 6 months be exclusively breastfed, many infants are also fed with other liquids such as water, non-milk liquids, and other milks before 6 months. Because of level of education and proportion of employed women in Ethiopia has been increasing gradually. Consequently the practice of exclusive breastfeeding became very lower.

The main objective of this study was to assess the magnitude and factors associated with the practice of exclusive breastfeeding among employed and unemployed mothers in Ethiopia, using dataset from EDHS 2016. 
METHOD: Data was extracted from Ethiopia Demographic and Health Survey (EDHS-2016). A total of 1089 mothers with infants aged 0 to 6 months in the data set were included in the analysis. Descriptive analysis, chi-square and binary logistic regression models were used. RESULT: This study has demonstrated a 64.7\%, 95\%CI(58.9-70.5) and 75.4\%, 95\%CI(72.378.2) prevalence of EBF practices among employed and unemployed mothers respectively in the $24 \mathrm{~h}$ preceding the survey. Other factors associated with EBF practice included being unemployed mother, larger to normal birth weight; infant aged 0-3 month old, infant being male, have radio in the house, infant being wanted, delivery by non CS mode and presence of ANC follow up during pregnancy were found to be significantly associated with exclusive breastfeeding practice.

Conclusion: the prevalence of exclusive breast feeding practice among employed mothers was significantly lower than that of unemployed mothers. Thus, Having in consideration the impact of appropriate infant and young children feeding practice on children's nutritional status and mortality rate, policy makers still need to give more emphasis on promotion of exclusive breastfeeding through creating an enabling environment targeting the extension of postnatal maternity leave up to the first six month.

Key words: Exclusive breastfeeding, maternal employment, EDHS, Ethiopia.

INTRODUCTION: World health organization(WHO) recommended that babies should be exclusively breastfed from birth until 6 months of age and thereafter continue breastfeeding with appropriate and sufficient complementary food(1). Breastfeeding is among the most effective ways to protect maternal and child health and promote healthy growth and optimal development in early childhood. Empowering and enabling women to breastfeed should be at the heart of countries' efforts to keep every child alive and to build healthy, smart and productive societies(2). In spite of what is known about the WHO recommendation and benefits of exclusive breast feeding for children, mothers, families and society, practice of exclusive breastfeeding is unsatisfactory in many parts of the world. Globally, rates of breastfeeding are far lower than it is expected. Less than half of newborns begin breastfeeding in the first hour after birth. Nearly $40 \%$ 57 of infants less than 6 months of age are exclusively breastfed, which is far from the target of 58 Sustainable Development Goal 2030 agenda(70\%)(3). The prevalence of exclusive breast 59 feeding $(\mathrm{EBF})$ is low in lower and middle income country (LMIC). It was 37\% in 2016. The 60 overall prevalence of EBF in sub Saharan Africa (SSA) was 36.0\%, the prevalence was highest 
61 in Rwanda and lowest in Gabon(4). According to maternal and child survival program (MCSP)

62 Ethiopia ranked first by prevalence of early introduction of foods and liquids among Low- and

63 Middle-Income Countries(5). In Ethiopian Contrary to the recommendation, children under the

64 age of 6 months be exclusively breastfed, many infants are also fed with other liquids such as

65 water, non-milk liquids, and other milks before reaching age 6 months. In this report, 11\% of

66 infants begin complementary foods before 6 months of age, with more than one-fifth of children

67 consuming complementary foods by age 4-5 months(6). This inappropriate breastfeeding

68 practices during infancy, and childhood expose them to malnutrition. The malnutrition

69 contributes as underlying factor for the two top fatal diseases(diarrhea and pneumonia) among

70 infant and children(7).

71 Different studies indicated that the low prevalence of EBF practice in most developing countries

72 including Ethiopia is associated with many factors such as: socio-demographic, obstetric, health

73 service and employment related factors $(6,8-10)$. Returning to work after giving birth is a major

74 change for a woman and in her family's life. Returning to work while still breastfeeding is even

75 more of a challenge and it is one of the main reasons that women stop breastfeeding(11). Studies

76 in Ethiopia indicate that significant difference (10-30\%) was observed between employed and

77 unemployed mothers on practice of exclusive breastfeeding and the improvement on EBF is

$78 \operatorname{low}(12)(13)(14,15)$.

79 But there is limited studies that determine magnitude and factors associated with exclusive breast

80 feeding among employed and non-employed mothers even all of these studies are constrained to

81 town/community within one zone or districts. In this regard, there is a need to study this issue among

82 the employed and unemployed mothers at national level to come up with strong evidence on the

83 difference of their practice and to investigate and identify factors associated with EBF. Findings

84 from this study help child bearing mothers and their child by improving exclusive breastfeeding

85 and enable policy makers, stakeholders and public health researchers to develop interventions to

86 improve exclusive breastfeeding in the country. 
Data source

This study used dataset from the EDHS 2016. The survey was conducted by the central Statistical Agency (CSA) along with the Federal Ministry of Health (FMoH) and the Ethiopian Public Health Institute (EPHI) from January 18, 2016 to June 27, 2016.

92 Study design

93 The current study design was a comparative cross-sectional study using the dataset. Practice and 94 factors affecting the practice of EBF were compared between employed and unemployed mothers who have infants 6 month or less in the dataset.

Participants of current study

97 In EDHS 2016, 8 different datasets were used to categorize data. For current study data was extracted from kid's dataset. Women 15-49 years old with children six months or less old in the dataset were considered as the respondent.

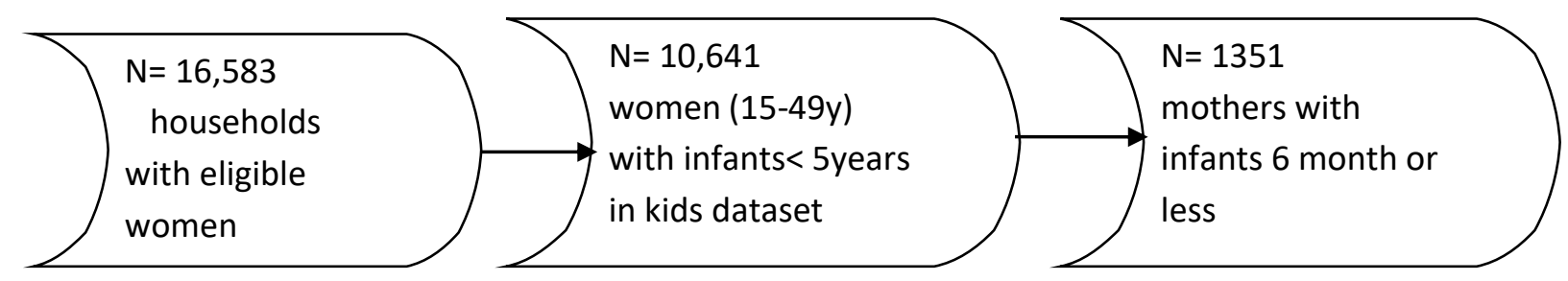

Figure 2: Selection of Study Sample

104

105

106

107

108

\section{Exclusion and inclusion criteria}

Mothers with children aged six months or less in the dataset were included as the study population. Children who did not live with their mother, those children's who had incomplete information about last 24 hour feeding practice, children whose mothers occupational status was incomplete in the dataset, and mothers who were working in private business such as merchant and agricultural work were excluded from the study.

\section{Data extraction}

Data was extracted from kids' dataset in the EDHS 2016 using data extraction sheet. Data extraction sheet contained socio-demographic characteristics, household characteristics, infant characteristics, obstetric and health service related characteristics and exclusive breast feeding practice. 
Dependant variable: practice of exclusive breastfeeding.

Independent Variables: Selection and categorization of independent variables in this study was based on literature review and availability of the variables. Socio-demographic characteristics of the mother and father, household characteristics, infant characteristics, obstetric and health service related characteristics $(9,16-19)$.

\section{The operational definition}

Exclusive breastfeeding: EBF was defined as feeding only breast milk (including milk expressed or from a wet nurse) without anything else in the last $24 \mathrm{~h}$ preceding the interview except for ORS, drops, and syrups (vitamins, minerals, medicines) for therapeutic purposes(20)

Employed mothers: Mothers who were working full time in either governmental organization or NGOs by the time of data collection were considered as employed mothers(21).

Unemployed mothers: mothers who were housewives (without a job) were considered as unemployed in this study (21).

\section{Data analysis}

First dataset was downloaded from DHS center after registration and permission obtained. Then the analysis was performed on the basis of children whose age is six months or less; alive and living with their mother at the dataset that was extracted from the kid's recode dataset. To estimate the magnitude of exclusive breastfeeding practice, the proportion of women (with infants aged between 0 and 6 months) who stated to have fed their children only breast milk in the last 24-hours preceding the survey in the dataset, were expressed as an EBF percentage of the total number of children in the age group of 0-6 month. The other independent variables applicable to this study in the dataset were transformed and recoded in to category if important. Descriptive analysis was carried out to examine the distribution of each individual variable. Then, chi-square test was done to compare the distribution of independent variables between employed and unemployed mothers with p-value 0.05. Bivariate analysis was carried out to describe association between pairs of variables. Finally, factors which were significant for bivariate association were observed with p-value 0.25 and retained for subsequent multivariate analyses using logistic regressions to control for possible confounders. Odds ratios were used to 
measure the strength of the association between dependent and independent variables. 95\% CI and p-value was used to determine the significance of the associations. Data analysis was done using the SPSS (version 23). Findings was presented by use of frequency distribution, tables, charts and texts.

\section{Ethics}

Permission to use and analyze the dataset was obtained by registering on the Demographic and Health Survey (DHS) website.

\section{Dissemination of Finding}

The finding of this study was presented and submitted to Arba minch University health Science College and public health department. Attempt will be made to Disseminate and present the findings to the $\mathrm{FMOH}$, different organizations that will have a contribution to improve the status of EBF in the Ethiopia. Various seminars and workshops, different journals and DHS center.

\section{RESULT}

\section{Socio-Demographic Characteristics}

The mean (SD) age of employed and unemployed mothers was 28(6.1) and 27(6.4) years respectively. Regarding to the educational status of mother, about 66(27.4\%) of employed and $418(49.3 \%)$ of unemployed mothers were illiterate. More than half of employed 140(58.1\%) and majority $(82.8 \%)$ of unemployed mothers were residing in rural area. About forty one percent of employed and 61.7\% unemployed participants were Muslim followed by orthodox. Among employed mothers nearly half (49.4\%) of their children were in the age group 0-3 months, while among unemployed mothers four hundred seventy one $(55.5 \%)$ of children were in the age group 0-3 months. Regarding to house hold wealth index, 52(21.6\%) of employed mothers and $359(42.3 \%)$ of unemployed mothers were from poorest family. Among employed mothers about $45(18.7 \%)$ of mothers had three or more under five children while among unemployed mothers $233(27.5 \%)$ had such much children. One hundred sixteen $(48.1 \%)$ of employed mothers perceived their child average in size during birth but it was 333(39.3\%) among unemployed mothers. About $68(28.2 \%)$ of employed mothers had TV in their house but only 112(13.2\%) of unemployed mothers had TV in their house 
Table 1: Socio-demographic characteristics in Ethiopia, 2016

\begin{tabular}{|c|c|c|c|c|c|c|}
\hline Variables & Category & $\begin{array}{c}\text { Employed } \\
\mathrm{N}=241(22 \%)\end{array}$ & $\begin{array}{l}\text { Unemployed } \\
\mathrm{N}=\mathbf{8 4 8}(\mathbf{7 8 \%})\end{array}$ & $\begin{array}{c}\text { Total } \\
\text { N=1089(100\%) }\end{array}$ & $\begin{array}{c}\mathrm{X}^{2} \\
\text { value }\end{array}$ & $\begin{array}{c}\mathbf{P} \\
\text { value }\end{array}$ \\
\hline \multirow{6}{*}{$\begin{array}{c}\text { women's age in } \\
\text { year }\end{array}$} & $15-19$ & $18(7.5 \%)$ & $85(10 \%)$ & $103(9.5 \%)$ & \multirow[t]{6}{*}{9.4} & \multirow[t]{6}{*}{0.093} \\
\hline & $20-24$ & $56(23.2 \%)$ & $246(29.0 \%)$ & $302(27.7 \%)$ & & \\
\hline & $25-29$ & $80(33.2 \%)$ & $229(27.0 \%)$ & $309(28.4 \%)$ & & \\
\hline & $30-34$ & $53(22 \%)$ & $145(17.1 \%)$ & $198(18.2 \%)$ & & \\
\hline & $35-39$ & $27(11.2 \%)$ & $113(13.3 \%)$ & $140(12.9 \%)$ & & \\
\hline & $40-49$ & $7(2.9 \%)$ & $30(3.5 \%)$ & $37(3.4 \%)$ & & \\
\hline \multirow{4}{*}{$\begin{array}{c}\text { husband/partner } \\
\text { 's age }\end{array}$} & $<20$ & $4(1.7 \%)$ & $21(2.5 \%)$ & $25(2.3 \%)$ & \multirow[t]{4}{*}{3.1} & \multirow[t]{4}{*}{0.377} \\
\hline & $21-40$ & $197(81.7 \%)$ & $660(77.8 \%)$ & $857(78.7 \%)$ & & \\
\hline & $41-60$ & $33(13.7 \%)$ & $149(17.6 \%)$ & $182(16.7 \%)$ & & \\
\hline & $>60$ & $7(2.9 \%)$ & $18(2.1 \%)$ & $25(2.3)$ & & \\
\hline \multirow{2}{*}{$\begin{array}{l}\text { educational } \\
\text { status of the }\end{array}$} & Illiterate & $93(38.6 \%)$ & $532(62.7 \%)$ & $625(57.4 \%)$ & \multirow[t]{4}{*}{73.3} & \multirow[t]{4}{*}{$<0.001$} \\
\hline & Primary & $79(32.8 \%)$ & $229(27.0 \%)$ & $308(28.3 \%)$ & & \\
\hline father or & Secondary & $43(17.8 \%)$ & $69(8.1 \%)$ & $112(10.3 \%)$ & & \\
\hline husband & Higher & $26(10.8 \%)$ & $18(2.1 \%)$ & $44(4.0 \%)$ & & \\
\hline \multirow{4}{*}{$\begin{array}{c}\text { educational } \\
\text { status of the } \\
\text { mother }\end{array}$} & $\begin{array}{l}\text { No } \\
\text { education }\end{array}$ & $66(27.4 \%)$ & $418(49.3 \%)$ & $484(44.4 \%)$ & \multirow[t]{4}{*}{50.8} & \multirow[t]{4}{*}{$<0.001$} \\
\hline & Primary & $92(38.2 \%)$ & $273(32.2 \%)$ & $365(33.5 \%)$ & & \\
\hline & Secondary & $39(16.2 \%)$ & $98(11.6)$ & $137(12.6 \%)$ & & \\
\hline & Higher & $44(18.3 \%)$ & $59(7 \%)$ & $103(9.5 \%)$ & & \\
\hline
\end{tabular}

173 Table 1 continued....................

\begin{tabular}{ccccccc}
\hline Variables & Category & $\begin{array}{c}\text { Employed } \\
\mathrm{N}=241(22 \%)\end{array}$ & $\begin{array}{c}\text { Unemployed } \\
\mathrm{N}=848(78 \%)\end{array}$ & $\begin{array}{c}\text { Total } \\
\mathrm{N}=1089(100 \%)\end{array}$ & $\begin{array}{c}\mathrm{X}^{2} \\
\text { value }\end{array}$ & $\begin{array}{c}\mathrm{P} \\
\text { value }\end{array}$ \\
\hline current marital & Married & $225(93.4 \%)$ & $812(95.8 \%)$ & $1037(95.2 \%)$ & 2.4 & 0.124 \\
status of mother & Others & $16(6.6 \%)$ & $36(4.2 \%)$ & $52(4.8 \%)$ & & \\
Type of place of & Urban & $101(41.9 \%)$ & $146(17.2 \%)$ & $247(22.7 \%)$ & 65.2 & $<0.001$ \\
residence & Rural & $140(58.1 \%)$ & $702(82.8 \%)$ & $842(77.3 \%)$ & & \\
& Orthodox & $93(38.6)$ & $168(19.8 \%)$ & $261(24 \%)$ & 42.8 & $<0.001$ \\
Parental Religion & Muslim & $98(40.7 \%)$ & $524(61.8 \%)$ & $622(57.1 \%)$ & & \\
\hline
\end{tabular}




\begin{tabular}{|c|c|c|c|c|c|c|}
\hline & Other & $50(20.7 \%)$ & $156(18.4 \%)$ & $206(18.9 \%)$ & & \\
\hline \multirow{4}{*}{$\begin{array}{l}\text { Preceding birth } \\
\text { interval in } \\
\text { (months) }\end{array}$} & $<12$ & $31(12.9 \%)$ & $94(11.1 \%)$ & $125(11.5 \%)$ & \multirow[t]{4}{*}{6.1} & \multirow[t]{4}{*}{0.109} \\
\hline & $13-24$ & $36(14.9 \%)$ & $154(18.2 \%)$ & $190(17.4 \%)$ & & \\
\hline & $25-60$ & $131(54.4 \%)$ & $494(58.3 \%)$ & $625(57.4 \%)$ & & \\
\hline & $\geq 61$ & $43(17.8 \%)$ & $106(12.5 \%)$ & $149(13.7 \%)$ & & \\
\hline \multirow[t]{2}{*}{ sex of child } & Male & $113(46.9 \%)$ & $433(51.1 \%)$ & $546(50.1 \%)$ & \multirow[t]{2}{*}{1.3} & 0.253 \\
\hline & Female & $128(53.1 \%)$ & $415(48.9 \%)$ & $543(49.9 \%)$ & & \\
\hline \multirow{2}{*}{$\begin{array}{l}\text { current age of } \\
\text { child in months }\end{array}$} & $0-3$ & $119(49.4 \%)$ & $471(55.5 \%)$ & $590(54.2 \%)$ & \multirow[t]{2}{*}{2.9} & 0.090 \\
\hline & $4-6$ & $122(50.6 \%)$ & $377(44.5 \%)$ & $499(45.8 \%)$ & & \\
\hline \multirow{4}{*}{$\begin{array}{l}\text { Number of } \\
\text { household } \\
\text { members }\end{array}$} & $1-3$ & $24(10.0 \%)$ & $120(14.2 \%)$ & $144(13.2 \%)$ & \multirow[t]{4}{*}{5.6} & 0.131 \\
\hline & $4-6$ & $133(55.2 \%)$ & $405(47.8 \%)$ & $538(49.4 \%)$ & & \\
\hline & $7-9$ & $72(29.9 \%)$ & $266(31.4 \%)$ & $338(31.0 \%)$ & & \\
\hline & $\geq 10$ & $12(5.0 \%)$ & $57(6.7 \%)$ & $69(6.3 \%)$ & & \\
\hline \multirow{3}{*}{$\begin{array}{l}\text { Under } 5 \text { children } \\
\text { in household }\end{array}$} & 1 & $96(39.8 \%)$ & $278(32.8 \%)$ & $374(34.3 \%)$ & \multirow[t]{3}{*}{8.6} & 0.014 \\
\hline & 2 & $100(41.5 \%)$ & $337(39.8 \%)$ & $437(40.1 \%)$ & & \\
\hline & $\geq 3$ & $45(18.7 \%)$ & $233(27.5 \%)$ & $278(25.5 \%)$ & & \\
\hline \multirow{2}{*}{$\begin{array}{c}\text { Sex of household } \\
\text { Head }\end{array}$} & Male & $187(77.6 \%)$ & $671(79.1 \%)$ & $858(78.8 \%)$ & \multirow[t]{2}{*}{0.3} & 0.605 \\
\hline & Female & $54(22.4 \%)$ & $177(20.9 \%)$ & $231(21.2 \%)$ & & \\
\hline
\end{tabular}

Table 1 continued

\begin{tabular}{ccccccc}
\hline Variables & Category & $\begin{array}{c}\text { Employed } \\
\mathrm{N}=241(22 \%)\end{array}$ & $\begin{array}{c}\text { Unemployed } \\
\mathrm{N}=848(78 \%)\end{array}$ & $\begin{array}{c}\text { Total } \\
\mathrm{N}=1089(100 \%)\end{array}$ & $\begin{array}{c}\mathrm{X}^{2} \\
\text { value }\end{array}$ & P value \\
\hline House hold & Poorest & $52(21.6 \%)$ & $359(42.3 \%)$ & $411(37.7 \%)$ & 83.3 & $<0.001$ \\
wealth index & Poorer & $26(10.8 \%)$ & $131(15.5 \%)$ & $157(14.4 \%)$ & & \\
& Middle & $29(12.0 \%)$ & $100(11.8 \%)$ & $129(11.8 \%)$ & & \\
& Richer & $25(10.4 \%)$ & $106(12.5 \%)$ & $131(12.0 \%)$ & & \\
& Richest & $109(45.2 \%)$ & $152(17.9 \%)$ & $261(24.0 \%)$ & & \multirow{2}{*}{2.9} \\
Birth weight & LBW(<2.5) & $48(19.9 \%)$ & $207(24.4 \%)$ & $255(23.4 \%)$ & & \\
\cline { 2 - 5 } of infant & NBW(2.5-4) & $173(71.8 \%)$ & $560(66 \%)$ & $733(67.3 \%)$ & & \\
\hline
\end{tabular}




\begin{tabular}{|c|c|c|c|c|c|c|}
\hline & $\mathrm{HBW}(>4)$ & $20(8.3 \%)$ & $81(9.6 \%)$ & $101(9.3 \%)$ & & \\
\hline Perceived & Very large & $39(16.2 \%)$ & $113(13.3 \%)$ & $152(14.0 \%)$ & 12.0 & 0.017 \\
\hline \multirow[t]{4}{*}{$\begin{array}{c}\text { Size of child } \\
\text { at Birth }\end{array}$} & $\begin{array}{c}\text { Larger than } \\
\text { average }\end{array}$ & $21(8.7 \%)$ & $114(13.4 \%)$ & $135(12.4)$ & & \\
\hline & Average & $116(48.1 \%)$ & $333(39.3 \%)$ & $449(41.2 \%)$ & & \\
\hline & $\begin{array}{c}\text { Smaller than } \\
\text { average }\end{array}$ & $26(10.8 \%)$ & $94(11.1 \%)$ & $120(11.0 \%)$ & & \\
\hline & Very small & $39(16.2 \%)$ & $194(22.9 \%)$ & $233(21.4 \%)$ & & \\
\hline \multirow{2}{*}{$\begin{array}{l}\text { Household } \\
\text { has: TV }\end{array}$} & No & $173(71.8 \%)$ & $736(86.8 \%)$ & $909(83.5 \%)$ & 30.6 & $<0.001$ \\
\hline & Yes & $68(28.2 \%)$ & $112(13.2 \%)$ & $180(16.5 \%)$ & & \\
\hline Household & No & $150(62.2 \%)$ & $637(75.1 \%)$ & $787(72.3 \%)$ & 15.5 & $<0.001$ \\
\hline has: radio & Yes & $91(37.8 \%)$ & $211(24.9 \%)$ & $302(27.7 \%)$ & & \\
\hline \multirow{3}{*}{$\begin{array}{c}\text { Birth order of } \\
\text { infant }\end{array}$} & 1 & $61(25.3 \%)$ & $193(22.8 \%)$ & $254(23.3 \%)$ & 3.0 & 0.229 \\
\hline & $2-5$ & $135(56.0)$ & $453(53.4 \%)$ & $588(54.0 \%)$ & & \\
\hline & $>5$ & $45(18.7)$ & $202(23.8 \%)$ & $247(22.7 \%)$ & & \\
\hline
\end{tabular}

\section{Prevalence of exclusive breastfeeding practice}

179 The prevalence exclusive breast feeding practice among employed 156(64.7\%), 95\%CI (58.970.5) mothers was lower than that of unemployed mothers 639(75.4\%), 95\%CI (72.3-78.2) (P-

181 Value $=0.001$ ). The percentage of employed mother (77.6) who initiated breast feeding within 182 one hour after birth was higher than that of unemployed mother $(70.2)(p=0.049)$.

Table 2: prevalence of practice of exclusive breast feeding practice in Ethiopia, 2016

$\begin{array}{lllllll}\text { Variables } & \text { Category } & \text { Employed } & \text { Unemployed } & \text { Total } & \mathbf{X}^{\mathbf{2}} & \text { P value }\end{array}$
$\mathrm{N}=241(22 \%) \quad \mathrm{N}=848(78 \%) \quad \mathrm{N}=1089(100 \%) \quad$ value

\begin{tabular}{ccrcccc}
\hline $\begin{array}{c}\text { When child } \\
\text { put to breast }\end{array}$ & $\begin{array}{c}\text { Within } \\
\text { first hour }\end{array}$ & $187(77.6 \%)$ & $595(70.2 \%)$ & $782(71.8 \%)$ & 6.0 & 0.049 \\
$\begin{array}{c}1 \text { st time after } \\
\text { birth }\end{array}$ & after & $37(15.4 \%)$ & $188(22.2 \%)$ & $225(20.7 \%)$ & & \\
& Hours & & & & & \\
EBF & after Days & $17(7.1 \%)$ & $65(7.7 \%)$ & $82(7.5 \%)$ & & \\
\hline
\end{tabular}




\begin{tabular}{ccccc}
\hline $\begin{array}{c}\text { PRACTICE } \\
\text { for last 24hrs }\end{array}$ & No & $85(35.3 \%)$ & $209(24.6 \%)$ & $294(27 \%)$ \\
\hline
\end{tabular}

\section{Obstetric and health service related factors}

About $143(59.3 \%)$ of employed and more than three fourth of unemployed $652(76.8 \%)$ mothers were not informed about breastfeeding by health provider during their pregnancy or postnatal care. About 196(81.3\%) of the employed and 533(62.8\%) unemployed mothers have ANC visit by health professional in their last pregnancy. The proportion of employed mothers $145(60.2 \%)$ who give birth of their index child in health facility was higher than that of unemployed mothers $312(36.8 \%)$. About 30(3.5\%) of unemployed mothers give birth of their last child by caesarean section while it was $16(6.6 \%)$ among employed mothers. The percentage of employed mothers 28(11.6\%) who have postnatal care within 2 months of their birth was higher than that of unemployed mothers 51(4.8\%).

Table 3: Obstetric and health service related factors by employment status in Ethiopia, 2016

\begin{tabular}{|c|c|c|c|c|c|c|}
\hline Variables & Category & $\begin{array}{c}\text { Employed } \\
\mathrm{N}=241(22 \%)\end{array}$ & $\begin{array}{l}\text { Unemployed } \\
\mathrm{N}=\mathbf{8 4 8}(\mathbf{7 8 \%})\end{array}$ & $\begin{array}{c}\text { Total } \\
\mathrm{N}=1089(100 \%)\end{array}$ & $\begin{array}{r}\mathrm{X}^{2} \\
\text { value }\end{array}$ & $P$ value \\
\hline \multirow[t]{3}{*}{$\begin{array}{c}\text { Wanted last } \\
\text { Child }\end{array}$} & $\begin{array}{l}\text { Wanted } \\
\text { then }\end{array}$ & $189(78.4 \%)$ & $714(84.2 \%)$ & $903(82.9 \%)$ & \multirow[t]{3}{*}{5.3} & \multirow[t]{3}{*}{0.072} \\
\hline & $\begin{array}{c}\text { Wanted } \\
\text { later }\end{array}$ & $36(14.9 \%)$ & $101(11.9 \%)$ & $137(12.6 \%)$ & & \\
\hline & $\begin{array}{l}\text { Wanted } \\
\text { no more }\end{array}$ & $16(6.6 \%)$ & $33(3.9 \%)$ & $49(4.5 \%)$ & & \\
\hline ANC by & No & $45(18.7 \%)$ & $315(37.2 \%)$ & $360(33.1 \%)$ & \multirow[t]{2}{*}{28.9} & \multirow[t]{2}{*}{$<0.001$} \\
\hline $\begin{array}{c}\text { health } \\
\text { Professional }\end{array}$ & Yes & $196(81.3 \%)$ & $533(62.8 \%)$ & $729(66.9 \%)$ & & \\
\hline Place of & Home & $96(39.8)$ & $536(63.2 \%)$ & $632(58.0 \%)$ & \multirow[t]{2}{*}{42.1} & \multirow[t]{2}{*}{$<0.001$} \\
\hline Delivery & $\begin{array}{l}\text { Health } \\
\text { facility }\end{array}$ & $145(60.2 \%)$ & $312(36.8 \%)$ & $457(42.0 \%)$ & & \\
\hline
\end{tabular}




\begin{tabular}{|c|c|c|c|c|c|c|}
\hline Health & No & $143(59.3 \%)$ & $652(76.8 \%)$ & $795(73.0 \%)$ & \multirow[t]{2}{*}{29.3} & \multirow[t]{2}{*}{$<0.001$} \\
\hline $\begin{array}{c}\text { provider } \\
\text { counsel on } \\
\text { BF }\end{array}$ & Yes & $98(40.7 \%)$ & $196(23.2 \%)$ & $294(27.0 \%)$ & & \\
\hline Last birth & No & $225(93.4 \%)$ & $818(96.5 \%)$ & $1043(95.8 \%)$ & \multirow[t]{2}{*}{4.5} & \multirow[t]{2}{*}{0.035} \\
\hline $\begin{array}{c}\text { caesarean } \\
\text { Section }\end{array}$ & Yes & $16(6.6 \%)$ & $30(3.5 \%)$ & $46(4.2 \%)$ & & \\
\hline $\begin{array}{c}\text { Timing of } \\
\text { 1st antenatal }\end{array}$ & $\begin{array}{c}\text { 0-4 } \\
\text { month }\end{array}$ & $140(58.1 \%)$ & $312(36.8 \%)$ & $452(41.5 \%)$ & \multirow[t]{3}{*}{40.5} & \multirow[t]{3}{*}{$<0.001$} \\
\hline check & $\begin{array}{c}5-6 \\
\text { month }\end{array}$ & $46(19.1 \%)$ & $171(20.2 \%)$ & $217(19.9 \%)$ & & \\
\hline & $\begin{array}{c}\text { 7-9 } \\
\text { month }\end{array}$ & $55(22.8 \%)$ & $365(43.0 \%)$ & $420(38.6 \%)$ & & \\
\hline
\end{tabular}

\begin{tabular}{|c|c|c|c|c|c|c|}
\hline Variables & Category & $\begin{array}{c}\text { Employed } \\
\mathrm{N}=241(22 \%)\end{array}$ & $\begin{array}{l}\text { Unemployed } \\
\mathrm{N}=848(78 \%)\end{array}$ & $\begin{array}{c}\text { Total } \\
\mathrm{N}=1089(100 \%)\end{array}$ & $\begin{array}{r}\mathrm{X}^{2} \\
\text { value }\end{array}$ & $P$ value \\
\hline Number of & No ANC & $45(18.7 \%)$ & $308(36.4 \%)$ & $353(32.4 \%)$ & \multirow[t]{4}{*}{31.5} & \multirow[t]{4}{*}{$<0.001$} \\
\hline ANC during & $1-2$ & $38(15.8 \%)$ & 139(16.4) & $177(16.3 \%)$ & & \\
\hline \multirow[t]{2}{*}{ pregnancy } & $3-4$ & $87(36.1 \%)$ & $240(28.3 \%)$ & $327(30.0 \%)$ & & \\
\hline & $\geq 5$ & $71(29.5 \%)$ & 161(18.9\%) & $232(21.3 \%)$ & & \\
\hline Respondent's & No & $222(92.1 \%)$ & $806(95.0 \%)$ & $1028(94.4 \%)$ & \multirow[t]{2}{*}{3.0} & \multirow[t]{2}{*}{0.081} \\
\hline $\begin{array}{l}\text { health } \\
\text { checked } \\
\text { after } \\
\text { discharge }\end{array}$ & Yes & $19(7.9 \%)$ & $42(5.0 \%)$ & $61(5.6 \%)$ & & \\
\hline postnatal & No & $213(88.4)$ & $797(95.2 \%)$ & $1010(92.7 \%)$ & \multirow[t]{2}{*}{8.7} & \multirow[t]{2}{*}{0.003} \\
\hline check & Yes & $28(11.6)$ & $51(4.8 \%)$ & $79(7.3 \%)$ & & \\
\hline
\end{tabular}


within 2

months

\section{Work related factors}

202 From the total mother-infant pairs, 241(22\%) mothers were employed and 848(78\%) were unemployed.

204

206

207

208

209

210

211

212

213

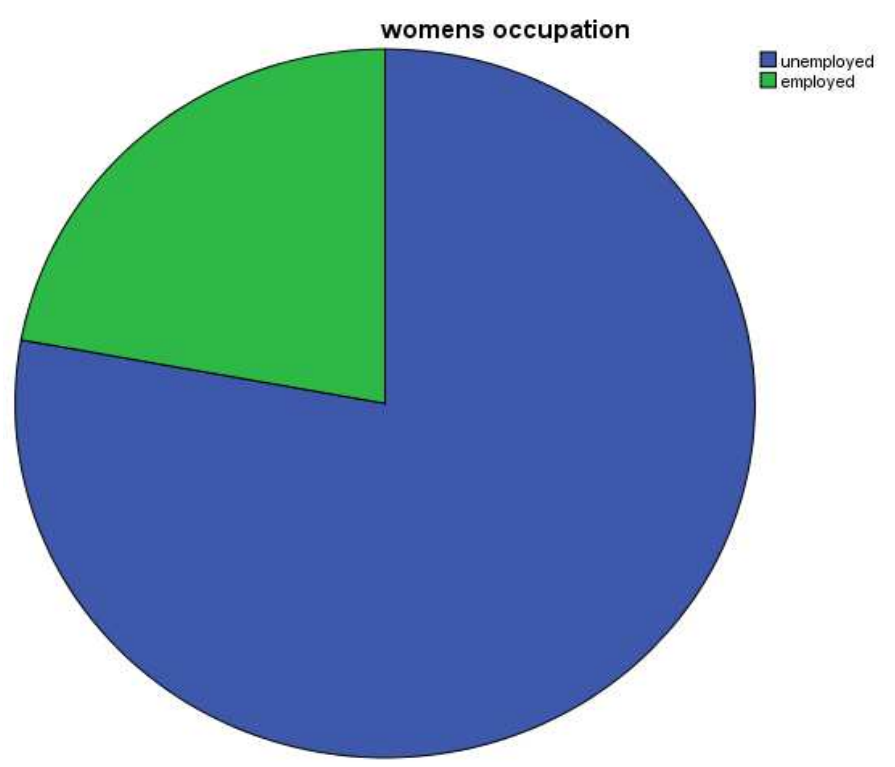

Figure 3: Percentage of employment status of mothers

Factors associated with exclusive breast feeding practice.

Variables that were significant in the bivariate analysis at $\mathrm{p}$ value 0.25 were retained for subsequent multivariate analysis. After controlling for other variables, being unemployed mother, being child wanted, younger age of infant, non CS mode of delivery, larger birth weight, presence of media access, infant being male and mothers had ANC follow up during pregnancy were significantly associated with EBF practice in the multivariate logistic regression model for the overall study participants. Among all, those mothers who were unemployed were 1.7 times more likely to practice EBF when compared to those of employed mothers (AOR=1.67, 95\%CI: 1.17-2.40). Mothers whose infants were larger to normal birth weight were $61 \%$ less likely to practice EBF compared to those of low birth weight [AOR=0.39, 95\%CI: 0.21-0.72]; Regarding to age of infants, mothers whose infant was 0-3 month old were 4.3 (AOR=4.27, 95\% CI: 3.145.81) times more likely to practice exclusive breast feeding than mothers whose child was 4-6 month old. Mothers whose child was male in their sex were $29 \%$ less likely to practice EBF than 
219 their counter parts $(\mathrm{AOR}=0.71,95 \% \mathrm{CI}$ : 0.53-0.96). Mothers who wanted their last pregnancy

220 later were 2.3 times more likely to practice EBF than mothers who never wanted their pregnancy $221(\mathrm{AOR}=2.29,95 \% \mathrm{CI}: 1.05-5.01)$. Mothers who give birth of their last child by non CS mode of 222 delivery were 2.2 times more likely to practice EBF than their counter parts ( $\mathrm{AOR}=2.22,95 \% \mathrm{CI}$ : 223 1.09-4.55). Mothers who have ANC follow up during their last pregnancy were 1.5 times more 224 likely to practice EBF than mothers who have no ANC follow up (AOR= 1.52, 95\%CI: 1.06225 2.18). Those Mothers who have radio in their house were 1.5 times more likely to practice EBF 226 when compared with their counter parts $(\mathrm{AOR}=1.53,95 \% \mathrm{CI}: 1.07-2.21)$.

Table 4: Multivariate logistic regression of factors associated with practice of exclusive breastfeeding among employed and unemployed mothers in Ethiopia, 2016

\begin{tabular}{|c|c|c|c|c|c|c|}
\hline \multirow[t]{2}{*}{ Variables } & \multirow[t]{2}{*}{ Category } & \multicolumn{2}{|c|}{$\begin{array}{c}\text { Exclusive } \\
\text { breastfeedin } \\
\text { g practice }\end{array}$} & \multirow[t]{2}{*}{$\mathrm{COR}(95 \% \mathrm{CI})$} & \multirow[t]{2}{*}{$\mathrm{AOR}(95 \% \mathrm{CI})$} & \multirow[t]{2}{*}{$\mathrm{P}$ value } \\
\hline & & Yes & No & & & \\
\hline \multirow{2}{*}{$\begin{array}{c}\text { Occupational } \\
\text { status of } \\
\text { mother }\end{array}$} & Unemployed & 639 & 209 & $1.67(1.23-2.26)^{* *}$ & 1.67(1.17-2.40) & $0.005 *$ \\
\hline & Employed & 156 & 85 & 1 & 1 & \\
\hline \multirow{3}{*}{$\begin{array}{l}\text { Birth weight } \\
\text { in } \\
\text { Kilograms }\end{array}$} & $\mathrm{LBW}(<2.5)$ & 199 & 56 & 1 & 1 & 0.011 \\
\hline & NBW(2.5-4) & 535 & 198 & $0.76(0.54-1.07)$ & $0.74(0.49-1.13)$ & 0.166 \\
\hline & $\mathrm{HBW}(>4)$ & 61 & 40 & $0.43(0.26-0.71) *$ & $0.39(0.21-0.72)$ & $0.003 *$ \\
\hline Perceived & Very large & 106 & 46 & $0.65(0.41-1.03)$ & $1.20(0.68-2.11)$ & 0.529 \\
\hline \multirow[t]{4}{*}{$\begin{array}{l}\text { Size of child } \\
\text { at birth }\end{array}$} & $\begin{array}{c}\text { Larger than } \\
\text { average }\end{array}$ & 95 & 40 & $0.67(0.41-1.08)$ & $1.60(0.59-1.90)$ & 0.847 \\
\hline & Average & 330 & 119 & $0.78(0.53-1.13)$ & $1.01(0.64-1.60)$ & 0.960 \\
\hline & $\begin{array}{c}\text { Smaller than } \\
\text { average }\end{array}$ & 82 & 38 & $0.61(0.37-0.99) *$ & $0.66(0.38-1.16)$ & 0.149 \\
\hline & Very small & 182 & 51 & 1 & 1 & 0.416 \\
\hline Current age & $0-3$ & 506 & 84 & $4.38(3.27-5.86)^{* * *}$ & 4.27(3.14-5.81) & $<0.001 * *$ \\
\hline of child in & $4-6$ & 289 & 210 & 1 & 1 & $<0.001$ \\
\hline
\end{tabular}




\begin{tabular}{|c|c|c|c|c|c|c|}
\hline months & & & & & & \\
\hline \multirow{2}{*}{$\begin{array}{l}\text { Type of place } \\
\text { of residence }\end{array}$} & Urban & 166 & 81 & 1 & \multicolumn{2}{|l|}{1} \\
\hline & Rural & 629 & 213 & $1.44(1.06,1.96)^{*}$ & $1.45(0.80-2.62)$ & 0.223 \\
\hline Wanted last & Wanted then & 663 & 240 & 2.07(1.16-3.72)* & $1.81(0.92-3.56)$ & 0.085 \\
\hline \multirow[t]{2}{*}{ Child } & Wanted later & 104 & 33 & $2.36(1.19-4.70) *$ & $2.29(1.05-5.01)$ & 0.038* \\
\hline & $\begin{array}{c}\text { Wanted no } \\
\text { more }\end{array}$ & 28 & 21 & 1 & 1 & 0.115 \\
\hline
\end{tabular}

229

230 Table 4: continued.................

\begin{tabular}{|c|c|c|c|c|c|c|}
\hline \multirow[t]{2}{*}{ Variables } & \multirow[t]{2}{*}{ Category } & \multicolumn{2}{|c|}{$\begin{array}{c}\text { Exclusive } \\
\text { breastfeeding } \\
\text { practice }\end{array}$} & \multirow[t]{2}{*}{$\mathrm{COR}(95 \% \mathrm{CI})$} & \multirow[t]{2}{*}{$\mathrm{AOR}(95 \% \mathrm{CI})$} & \multirow[t]{2}{*}{$\mathrm{P}$ value } \\
\hline & & Yes & No & & & \\
\hline \multirow{2}{*}{$\begin{array}{c}\text { Last birth } \\
\text { caesarean } \\
\text { Section }\end{array}$} & No & 772 & 271 & $2.85(2.48-3.27)^{*}$ & $2.22(1.09-4.55)$ & 0.029* \\
\hline & Yes & 23 & 23 & 1 & 1 & \\
\hline \multirow{3}{*}{$\begin{array}{l}\text { When child } \\
\text { put to breast }\end{array}$} & Immediately & 579 & 203 & $1.83\left((1.14-2.93)^{*}\right.$ & $1.42(0.83-2.41)$ & 0.198 \\
\hline & after Hours & 166 & 59 & $1.80(1.06-3.07)^{*}$ & $1.40(0.76-2.57)$ & 0.285 \\
\hline & after Days & 50 & 32 & 1 & 1 & 0.433 \\
\hline \multirow[t]{2}{*}{ Sex of child } & Male & 382 & 164 & $0.73(0.56-0.96)^{*}$ & $0.71(0.53-0.96)$ & $0.028 *$ \\
\hline & Female & 413 & 130 & 1 & 1 & \\
\hline \multirow{2}{*}{$\begin{array}{l}\text { Had ANC } \\
\text { follow up }\end{array}$} & No & 253 & 107 & 1 & 1 & \\
\hline & Yes & 542 & 187 & $1.23(0.93,1.62)$ & $1.52(1.06-2.18)$ & $0.022 *$ \\
\hline \multirow{4}{*}{$\begin{array}{l}\text { Educational } \\
\text { status of the } \\
\text { mother }\end{array}$} & $\begin{array}{c}\text { No } \\
\text { education }\end{array}$ & 371 & 113 & $1.69(1.07-2.67) *$ & $1.57(0.87-2.84)$ & 0.137 \\
\hline & Primary & 256 & 109 & $1.21(0.76-1.93)$ & $0.99(0.57-1.73)$ & 0.968 \\
\hline & Secondary & 100 & 37 & $1.39(0.80-2.42)$ & $1.23(0.66-2.31)$ & 0.516 \\
\hline & Higher & 68 & 35 & 1 & 1 & 0.093 \\
\hline \multirow{3}{*}{$\begin{array}{c}\text { Preceding } \\
\text { birth interval } \\
\text { in (months) }\end{array}$} & $<12$ & 86 & 39 & 1 & 1 & 0.062 \\
\hline & $13-24$ & 136 & 54 & $1.14(0.70,1.87)$ & $0.98(0.54-1.77)$ & 0.945 \\
\hline & $25-60$ & 457 & 168 & $1.23(0.81,1.87)$ & $0.98(0.59-1.64)$ & 0.941 \\
\hline
\end{tabular}




\begin{tabular}{|c|c|c|c|c|c|c|}
\hline \multirow{6}{*}{ Wealth index } & $61+$ & 116 & 33 & $1.59(0.93,2.74)$ & $1.88(0.99-3.59)$ & 0.054 \\
\hline & Poorest & 304 & 107 & $1.23(0.87-1.74)$ & $0.69(0.34-1.42)$ & 0.314 \\
\hline & Poorer & 120 & 37 & $1.41(0.90-2.22)$ & $0.78(0.36-1.70)$ & 0.528 \\
\hline & Middle & 93 & 36 & $1.12(0.70-1.79)$ & $0.64(0.29-1.40)$ & 0.259 \\
\hline & Richer & 96 & 35 & $1.19(0.75-1.90)$ & $0.52(0.25-1.10)$ & 0.087 \\
\hline & Richest & 182 & 79 & 1 & 1 & 0.465 \\
\hline & & & & & & \\
\hline \multicolumn{7}{|c|}{ Table 4: continued ............... } \\
\hline \multirow[t]{4}{*}{ Variables } & Category & \multirow{3}{*}{\multicolumn{2}{|c|}{$\begin{array}{c}\text { Exclusive } \\
\text { breastfeeding } \\
\text { practice }\end{array}$}} & $\mathrm{COR}(95 \% \mathrm{CI})$ & \multirow[t]{4}{*}{$\operatorname{AOR}(95 \% \mathrm{CI})$} & \multirow[t]{4}{*}{$\mathrm{P}$ value } \\
\hline & & & & & & \\
\hline & & & & & & \\
\hline & & Yes & No & & & \\
\hline \multirow{4}{*}{$\begin{array}{c}\text { Husband/partn } \\
\text { er's age }\end{array}$} & $<20$ & 16 & 9 & 1 & 1 & 0.117 \\
\hline & $21-40$ & 640 & 217 & $1.66(0.72,3.81)$ & $1.87(0.75-4.69)$ & 0.181 \\
\hline & $41-60$ & 123 & 59 & $1.17(0.49,2.81)$ & $1.22(0.46-3.23)$ & 0.695 \\
\hline & $>60$ & 16 & 9 & $1.00(0.32,3.17)$ & $1.41(0.39-5.10)$ & 0.598 \\
\hline \multirow{2}{*}{$\begin{array}{c}\text { Household } \\
\text { has: TV }\end{array}$} & No & 673 & 236 & 1 & 1 & \\
\hline & Yes & 122 & 58 & $0.74(0.52,1.04)$ & $0.67(0.37-1.24)$ & 0.206 \\
\hline Household & No & 563 & 224 & 1 & 1 & \\
\hline has: radio & Yes & 232 & 70 & $1.32(0.97,1.80)$ & $1.53(1.07-2.21)$ & $0.022 *$ \\
\hline Baby Had & No & 744 & 266 & 1 & 1 & \\
\hline $\begin{array}{l}\text { postnatal } \\
\text { check }\end{array}$ & Yes & 51 & 28 & $0.65(0.40,1.05)$ & $0.67(0.38-1.18)$ & 0.160 \\
\hline \multicolumn{3}{|c|}{ Hosmer and Lemeshow Test } & \multicolumn{2}{|c|}{ Chi-square =2.856 } & \multicolumn{2}{|c|}{$\mathrm{P}-$ value $=0.943$} \\
\hline \multicolumn{4}{|c|}{ *-Significance level of $<0.05$} & \multicolumn{3}{|c|}{$*_{*}^{*}$-Significance level of $<0.001$} \\
\hline
\end{tabular}

234 This study revealed that the prevalence of exclusive breastfeeding practice among employed and 235 unemployed mothers was $64.7 \%$ and $75.4 \%$ respectively. These difference was statistical 236 significant $(\mathrm{P}-\mathrm{Value}=0.001)$. This result was higher for both employed and unemployed mothers 237 as compared to the study conducted in Gondar among children aged 7-12 months found $20.9 \%$ 
of employed and $48 \%$ of unemployed mothers breastfed their children for six months exclusively(13). It was in line for unemployed mothers but higher for employed mothers as compared to the study conducted in Goba district the prevalence of exclusive breastfeeding was $33 \%$ and $73 \%$ among employed and unemployed mothers respectively (15). In a similar trade, unemployed mothers were more likely to exclusively breastfeed their infants as compared to those of employed mothers. This indicates that maternal employment is a hindrance for EBF. In agreement with this, studies from Ethiopia, (22-24), Bangladesh(25), Mexico(26), and Canada(27) reported that maternal employment has a negative effect on EBF. Many reasons can be stated for this association. In accordance with the Ethiopia Labor Proclamation, female workers are entitled to fully paid maternity leave of 120 days (30 days antenatal and 90 days postnatal) on recommendation of medical doctor. Hence employed mothers will have a maximum of three months to stay at home and breastfed their infants which don't fit with the recommended six months of EBF. This short maternity leave period successively influences them to introduce complementary feeding starting from the time they return to work(28). Another study(29) reported that, each week of maternity leave increased the duration of breastfeeding by almost one-half week. Besides, since there is no breastfeeding friendly environment like facilities for breastfeeding at workplaces, employed mothers couldn't take their infants to the workplace and breastfeed there $(30,31)$. Therefore, the summative effect of the stated reasons above, would compromise the rate of EBF among employed mothers.

This study revealed that child's age had significant association with EBF practice. Mothers with younger infant had high tendency to practice EBF. This finding was consistent with the studies conducted in other parts of Ethiopia (16-18, 24), Brazil (32), Nigeria (33) and Congo (34). The first possible reason for this association could be the postpartum rest in the first few months after delivery that obligates women to stay at home. This, as a result may encourage them to breastfeed their infant. The other explanation may be due to the perception that some mothers have, that breast milk alone is not enough as the infant gets older.

On the other hand, mothers who wanted to give birth of their child later were more likely to practice EBF than who never wanted in the present study. This is due to the fact that mothers who gave birth of unwanted child were psychological not prepared to do what is expected from as a mother to her child. Prenatal exclusive breastfeeding intention was a strong predictor of 
268

269

270

271

272

273

274

275

276

277

278

279

280

281

282

283

284

285

286

287

288

289

exclusive breastfeeding practice. Intentions to EBF were further affected by having an unplanned pregnancy $(35,36)$.

Birth weight of infant was negatively associated with EBF practice. The possible explanation for this was that most of the time microsomia children are delivered through cesarean section. Again the cesarean delivery further affects exclusive breast feeding practice. This finding is inconsistent with finding in Australia (37).

Mothers who give birth of their last child by non CS mode of delivery were found to practice EBF more likely than those who give birth of their last child by CS delivery. This is due to the fact that cesarean delivery affects exclusive breast feeding. This finding was in line with finding in Ethiopia (38) Bangladesh (25), and Palestine (39).

In present study mothers whose index child was male were less likely to practice EBF than their counter parts. This finding contradicts the finding in Egypt(40), and New York(41). The possible reason for this association was the cultural difference in different parts of Ethiopia that prefer sex in feeding.

In line with previous studies $(17,25)$, mothers who had radio in their house were more likely to practice EBF than their counter parts. This is due to that mothers who have media access to get information about EBF had more knowledge about EBF practice.

Consistent with earlier studies $(18,25,40,42)$ ANC follow up was an independent determinant affecting EBF practice. Mothers who had ANC follow up were more likely to practice excusive breast feeding than their counter parts. This could be due to the ANC programs that include breastfeeding counseling which in turn improves breastfeeding knowledge of mothers and motivates.

In conclusion, the prevalence of EBF practices among employed mothers was lower than that of unemployed mothers, indicating a significant difference between the two groups. Hence, maternal employment has a negative influence on EBF practices.

In addition, being unemployed mother, presence of ANC follow up, younger age of infant, presence of media access like radio, non cesarean mode of delivery, large birth weight of child, being wanted child and child sex being male were also significantly associated with EBF practice. 
297 Having in consideration the impact of appropriate infant and young children feeding practice on 298 children's nutritional status and mortality rate, policy makers still need to give more emphasis on 299 promotion of exclusive breastfeeding through creating an enabling environment targeting the 300 extension of postnatal maternity leave up to the first six month.

301 Government and non government organization should launch breastfeeding-friendly work 302 environment for working mothers by establishing work-site day care centers for infants in order 303 to promote of EBF.

304 Health personnel who are working in the clinics should advise mothers to have a spontaneous 305 vaginal delivery and should not insist on performing C/S delivery unless and otherwise 306 medically justified.

307 Awareness should be created in community or among mothers that breast milk alone is enough 308 for any nutritional requirements of infant for the first 6 months after birth.

309 Increasing media coverage regarding the awareness programs of exclusive breastfeeding should 310 be considered to improve EBF practice.

311 Maternal health clinics and health extension program should be strengthened throughout the 312 country so that more number of pregnant women and mothers can receive appropriate maternal 313 health service both at the community and institutional levels, such as, ANC follow up ,Family 314 planning.

\section{ABBREVIATIONS AND ACRONYMS}

ANC

CSA

DHS

EA

EBF

EDHS

EPHI

FMoH

LMIC
Antenatal care

Central Statistical Agency

Demographic and Health Surveys

enumeration areas

Exclusive Breast Feeding

Ethiopia Demographic and Health Surveys

Ethiopian Public Health Institute

Federal Ministry of Health

lower and middle income country 
MCSP

PHC

SSA maternal and child survival program

Population and Housing Census

Sub Saharan Africa

\section{Availability of data and materials;}

The dataset supporting the conclusions of this article is included within the article.

\section{Supporting information;}

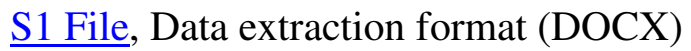

\section{Declaration of interests}

I declare no competing interests.

\section{Funding;}

The research was conducted fully independent from the study sponsor.

Authors' contributions;

Conceptualization: Desta Markos, Eshetu Yisihak

Data curation: Desta Markos, Eshetu Yisihak

Formal analysis: Desta Markos, Eshetu Yisihak

Funding acquisition: Desta Markos, Eshetu Yisihak

Methodology: Desta Markos, Eshetu Yisihak

Project administration: Desta Markos, Eshetu Yisihak

Resources: Desta Markos, Eshetu Yisihak

Software: Desta Markos, Eshetu Yisihak

\section{ACKNOWLEDGMENTS}

The author's thank Demographic and Health Surveys (DHS) Program center in Washington for providing nationally representative dataset collected by Ethiopia Demographic and Health Survey (EDHS), 2016. 
352 1. ORGANIZATION WH. Global strategy for infant and young child feeding: the Optimal Duration of Exclusive Breastfeeding. . WORLD HEALTH ORGANIZATION. Geneva 2001.

354 2. UNICEF. Breastfeeding_A_Mothers_Gift_for_Every_Child. UNICEF FOR EVERY CHILD. 2018.

355 3. World health organization u. ENABLING WOMEN TO BREASTFEED THROUGH BETTER POLICIES AND PROGRAMMES: GLOBAL BREASTFEEDING SCORECARD, 2018. global breast feeding collective. 2018.

4. YALCIN SS, BERDE, A. S. \& YALCIN, S. Determinants of Exclusive Breast Feeding in sub-Saharan Africa: A Multilevel Approach Paediatr Perinat Epidemiol. 2016;30:439-49.

5. USAID. Addressing Barriers to Exclusive Breastfeeding: Evidence and Program Considerations for Low- and Middle-Income Countries: MCSP Nutrition Brief. maternal and child survival program available at wwwmcsprogramorg. 2017.

6. $\quad$ CSA Central Statistical Authority A.A E. Ethiopia Demographic and Health Survey ICF International, Calverton, Maryland, USA. 2016.

7. Tewodros Alemayehu JH, Dereje Habte. Determinants of exclusive breastfeeding practices in Ethiopia. EthiopJHealth Dev. 2009.

8. Abay Sefene DB WA, Tesfaye Taye. Determinants of Exclusive Breastfeeding Practice among Mothers of Children Age Less Than 6 Month in Bahir Dar City Administration, Northwest Ethiopia; A Community Based Cross Sectional Survey. Science Journal of Clinical Medicine 2013; 2, (No. 6, 2013, ):153-9.

9. Yalcin SS, Berde AS, Yalcin S. Determinants of Exclusive Breast Feeding in sub-Saharan Africa: A Multilevel Approach. Paediatric and perinatal epidemiology. 2016;30(5):439-49.

10. Aluisio J D BJ, Sankar Simon, H Murch, Neff Walker. Breastfeeding in the 21st century: Epidemiology, mechanisms, and lifelong effect. lancet 2016.

11. WHO U. Maternity Protection Resource Package Breastfeeding arrangements at work. International Labour Organization. 2012.

12. Xiaodong Cai1 TW, David W Brown. Global trends in exclusive breastfeeding. International Breastfeeding Journal 2012.

13. Chekol DA, Biks GA, Gelaw YA, Melsew YA. Exclusive breastfeeding and mothers' employment status in Gondar town, Northwest Ethiopia: a comparative cross-sectional study. International breastfeeding journal. 2017;12(1):27.

381 14. Abdulbasit Musa Seid MEY, Digsu Negese Koye,. Prevalence of Exclusive Breastfeeding Practices and associated factors among mothers in Bahir Dar city, Northwest Ethiopia: a community based crosssectional study International Breastfeeding Journal

available at: http://wwwinternationalbreastfeedingjournalcom/content/8/1/14. 2013. Factors associated with exclusive breastfeeding practices among mothers in Goba district, south east Ethiopia: a cross-sectional study. . International Breastfeeding Journal available at: http://wwwinternationalbreastfeedingjournalcom/content/7/1/17 2012.

16. Hoche S, Meshesha B, Wakgari N. Sub-Optimal Breastfeeding and Its Associated Factors in Rural Communities of Hula District, Southern Ethiopia: A Cross-Sectional Study. Ethiopian journal of health sciences. 2018;28(1):49-62.

17. Tamiru D, Belachew T, Loha E, Mohammed S. Sub-optimal breastfeeding of infants during the first six months and associated factors in rural communities of Jimma Arjo Woreda, Southwest Ethiopia. BMC public health. 2012;12:363.

18. Tariku A, Alemu K, Gizaw Z, Muchie KF, Derso T, Abebe SM, et al. Mothers' education and ANC visit improved exclusive breastfeeding in Dabat Health and Demographic Surveillance System Site, northwest Ethiopia. PloS one. 2017;12(6):e0179056. 
19. Yeneabat T, Belachew T, Haile M. Determinants of cessation of exclusive breastfeeding in Ankesha Guagusa Woreda, Awi Zone, Northwest Ethiopia: a cross-sectional study. BMC Pregnancy Childbirth. 2014;14:262.

20. UNICEF. Infant and Young Child Feeding 2011.

21. Krapp KM WJ. The Gale Encyclopedia of Children's Health. Google Schoolar. 2005.

22. Tadesse. Exclusive breastfeeding and maternal employment among mothers of infants from three to five months old in the Fafan zone, Somali regional state of Ethiopia: a comparative crosssectional study BMC public health. 2019.

23. Chekol DA BG, Gelaw YA, Melsew YA. . Exclusive breastfeeding and mothers' employment status in Gondar town, Northwest Ethiopia: a comparative cross-sectional study. . International breastfeeding journal. 2017;12(1):27.

24. Yeneabat $t$, belachew, t. \& haile, $m$ Determinants of cessation of exclusive breastfeeding in Ankesha Guagusa Woreda, Awi Zone, Northwest Ethiopia: a cross-sectional study. BMC pregnancy and childbirth. 2014;14 262.

25. Hossain M, Islam A, Kamarul T, Hossain G. Exclusive breastfeeding practice during first six months of an infant's life in Bangladesh: a country based cross-sectional study. BMC pediatrics. 2018;18(1):93.

26. Letilcia escobra TGDC. Breastfeeding and Maternal Employment: Results from Three National Nutritional Surveys in Mexico. Maternal and child health journal. 2014

27. Al-Sahab B LA, Feldman M, Tamim H. Prevalence and predictors of 6-month exclusive breastfeeding among Canadian women: a national survey BMC Pediatr

2010;10:20.

28. FDRO. E. FEDERAL NEGARIT GAZETTE OF THE FEDERAL DEMOCRATIC REPUBLIC OF ETHIOPIA: Federal Civil Servants Proclamation NO.1064/2017. . 2017.

29. Ghwass MMA AD. Prevalence and predictors of 6-month exclusive breastfeeding in a rural area in Egypt. . Breastfeed Med 2011:;6(4):191-6.

30. Asfaw MM AM, Kefene ZK. Factors associated with exclusive breastfeeding practices in Debre Berhan District, Central Ethiopia: a cross sectional community based study. . Int Breastfeed J 2015:;10(1):1.

31. Radhakrishnan S BS. Prevalence of exclusive breastfeeding practices among rural women in Tamil Nadu. International Journal of Health \& Allied Sciences 2012;1(2):64.

32. Ferreira H, Oliveira MF, Bernardo EBR, Almeida PC, Aquino PS, Pinheiro AKB. Factors Associated with Adherence to the Exclusive Breastfeeding. Ciencia \& saude coletiva. 2018;23(3):683-90.

33. Gayawan E, Adebayo SB, Chitekwe S. Exclusive breastfeeding practice in Nigeria: a bayesian stepwise regression analysis. Maternal and child health journal. 2014;18(9):2148-57.

34. Dhakal S, Lee TH, Nam EW. Exclusive Breastfeeding Practice and Its Association among Mothers of under 5 Children in Kwango District, DR Congo. International journal of environmental research and public health. 2017;14(5).

35. Balogun OO, Kobayashi S, Anigo KM, Ota E, Asakura K, Sasaki S. Factors Influencing Exclusive Breastfeeding in Early Infancy: A Prospective Study in North Central Nigeria. Maternal and child health journal. 2016;20(2):363-75.

36. de Jager E, Broadbent J, Fuller-Tyszkiewicz $M$, Skouteris $H$. The role of psychosocial factors in exclusive breastfeeding to six months postpartum. Midwifery. 2014;30(6):657-66.

37. Biro MA, Sutherland GA, Yelland JS, Hardy P, Brown SJ. In-hospital formula supplementation of breastfed babies: a population-based survey. Birth (Berkeley, Calif). 2011;38(4):302-10. 
443 38. LaykewoldElyas A, AmhaAdmasie, andEtagegnehuAssefa. Exclusive Breastfeeding Practice and 444 Associated Factors among Mothers Attending Private Pediatric and Child Clinics, Addis Ababa, Ethiopia:

445 A Cross-Sectional Study. International Journal of Pediatrics 2017.

446 39. Musmar SG, Qanadeelu S. Breastfeeding patterns among Palestinian infants in the first 6 months 447 in Nablus refugee camps: a cross-sectional study. Journal of human lactation : official journal of 448 International Lactation Consultant Association. 2012;28(2):196-202.

449 40. Al Ghwass MM, Ahmed D. Prevalence and predictors of 6-month exclusive breastfeeding in a 450 rural area in Egypt. Breastfeeding medicine : the official journal of the Academy of Breastfeeding 451 Medicine. 2011;6(4):191-6.

452 41. Soni S, Gupta A, Jacobs AJ. Exclusive breastfeeding rates in a multiethnic population at a 453 community hospital. The Journal of reproductive medicine. 2011;56(5-6):195-8.

454 42. Ogbo FA, Page A, Idoko J, Agho KE. Population attributable risk of key modifiable risk factors 455 associated with non-exclusive breastfeeding in Nigeria. BMC public health. 2018;18(1):247. 